\title{
A Sensitivity-Based Three-Phase Weather- Dependent Power Flow Approach for Networks with Local Controllers_-PART II: Case Studies
}

\author{
Evangelos E. Pompodakis*, Arif Ahmed ${ }^{\dagger}$, and Minas C. Alexiadis ${ }^{\ddagger}$ \\ **Department of Electrical and Computer Engineering, Aristotle University of Thessaloniki, Greece \\ ${ }^{\dagger}$ TUMCREATE, 1 CREATE Way, \#10-02 CREATE Tower, Singapore 138602 \\ Email: " bobodakis@hotmail.com, ${ }^{\dagger}$ arif.ahmed@tum-create.edu.sg, ${ }^{*}$ minalex@auth.com
}

\begin{abstract}
Power flow is an integral part of distribution system planning, monitoring, operation, and analysis. This twopart paper proposes a sensitivity-based three-phase weatherdependent power flow approach for accurately simulating distribution networks with local voltage controllers (LVC). This part II, firstly, presents simulation results of the proposed approach in an 8-Bus and 7-Bus network, which are validated using dynamic simulation. Secondly, simulation results for the IEEE 8500-node network are also presented. An extensive comparison is conducted between the proposed sensitivity-based approach and the other existing power flow approaches with respect to result accuracy and convergence speed. Moreover, the influence of weather and magnetic effects on the power flow results and the LVC states is also investigated. Simulation results confirm that the proposed sensitivity-based approach produces more accurate results than the existing approaches since it considers the actual switching sequence of LVCs as well as the weather and magnetic effects on the network. Moreover, the proposed algorithm exhibits accelerated convergence due to the usage of the sensitivity parameters, which makes it an important tool for distribution system analysis.
\end{abstract}

Index Terms-Distributed power generation, Magnetic effects, Power flow, Time delays, Local voltage controllers, Weather effects.

\section{INTRODUCTION}

$\mathrm{T}$ HE first part of this paper presents the theoretical development of the proposed sensitivity-based threephase weather-dependent power flow approach for distribution networks with LVCs. The proposed approach has four distinct characteristics: a) it considers the three-phase unbalanced nature of distribution systems, b) the operating state of LVCs is calculated using sensitivity parameters to accelerate the convergence speed of the algorithm, c) it considers the exact switching sequence of LVCs based on their reaction time delays, and d) the influence of weather variations on the power flow is also taken into consideration.

This second part complements the theoretical development of part I by presenting simulation results in a balanced 8-Bus and an unbalanced 7-Bus network as well as in the large IEEE 8500-node network.

The rest of this Part II is structured as follows: Section II validates the proposed algorithm against dynamic simulation

This research is co-financed by Greece and the European Union (European Social Fund- ESF) through the Operational Programme «Human Resources Development, Education and Lifelong Learning» in the context of the project "Strengthening Human Resources Research Potential via Doctorate Research - 2nd Cycle" (MIS-5000432), implemented by the State Scholarships Foundation (IKY). via MATLAB ${ }^{\circledR}$ Simulink. Moreover, a comparison between the proposed algorithm and two existing approaches is presented in the same section. In Section III, a simulation case study of the proposed algorithm is presented for the largescale IEEE 8500-node network under two distinct weather conditions. The simulation results presented highlight the importance of considering the weather and magnetic effects in power flow analysis since they can significantly affect the power flow results and LVC state estimation. Finally, Section IV concludes the paper.

\section{VALIDATION AND PERFormance of THE PROPOSED SENSITIVITY-BASED ALGORITHM}

First and foremost, the proposed algorithm is validated against dynamic simulation in MATLAB ${ }^{\circledR}$ Simulink using an 8-bus balanced and a 7-bus unbalanced network. In addition, the proposed approach is compared against the power flow methods of [1] $]^{*}$ and [3], with respect to result accuracy and convergence speed. All algorithms were coded and implemented in MATLAB ${ }^{\circledR}$. It should be noted that for the purpose of validation against the dynamic simulation, the weather-dependent impacts were neglected in this section.

\section{A) 8-Bus Balanced Network}

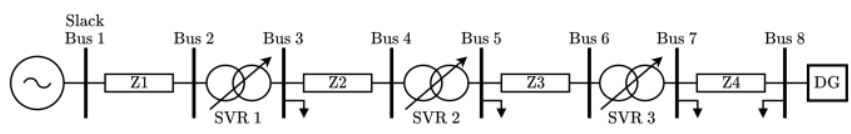

Fig. 1: 8-Bus network consisting of 3 SVRs and 1 DG.

Fig. 1 depicts the 8-Bus network consisting of 3 SVRs and 1 DG operating in $Q(V)$ droop control mode [4]. The $Q(V)$ droop equation is given by (1) [4]:

$$
V_{i}=V_{\text {ref(i) }}+K_{q(i)} \cdot Q_{i}
$$

* It is noted that the method proposed in [2] presents almost identical results with the method of [1]. Therefore, the method of [2] is not simulated in this paper due to its strong similarity with [1] with respect to the produced results. 
"This work has been submitted to IEEE for possible publication. Copyright may be transferred without notice, after which this version may no longer be accessible."

where $Q_{i}, V_{\text {ref(i) }}, K_{q(i)}, V_{i}$ are the positive sequence reactive power, the reference voltage, the droop gain, and the positive sequence voltage of DG $i$, respectively. The topology of the network is similar to the one investigated in [5]. Data about the network, the controllers of SVRs, and the DG are provided in Table I. The delays of the controllers are set based on their distance from the substation.

The slack bus in Fig 1 is assumed to be a substation. The SVR near the substation has the fastest reaction time and as the distance from the substation increases, the reaction time increases [6]. All SVRs are in wye configuration and initialized to the 0 position for the purposes of simulation. Each phase of an SVR is modelled with its own local controller, which is independently controlled.

The DG at bus 8 operates in $Q(V)$ mode and generates balanced phase-to-neutral voltages [7]. The network supplies four balanced three-phase loads. All the loads are modelled as constant impedance loads, as shown in Table II, due to the inaccuracy that Simulink presents in the modeling of constant power loads.

TABLE I

PARAMETERS OF 8-BUS NETWORK

\begin{tabular}{|c|c|}
\hline Distance of the lines & $10 \mathrm{~km}$ \\
\hline Voltage of slack bus & $7200 \mathrm{~V}$ \\
\hline Frequency of the network & $50 \mathrm{~Hz}$ \\
\hline Resistance of lines & $0.4 \Omega / \mathrm{km}$ \\
\hline Self-reactance of the lines & $0.3 \Omega / \mathrm{km}$ \\
\hline Mutual-reactance of the lines & $0.1 \Omega / \mathrm{km}$ \\
\hline Reference voltage of SVRs & $7500 \mathrm{~V}$ \\
\hline Bandwidth of SVRs & $70 \mathrm{~V}$ \\
\hline Intentional delay of SVR1 & $10 \mathrm{~s}$ \\
\hline Mechanical delay of SVR1 & $2 \mathrm{~s}$ \\
\hline Intentional delay of SVR2 & $20 \mathrm{~s}$ \\
\hline Mechanical delay of SVR2 & $3 \mathrm{~s}$ \\
\hline Intentional delay of SVR3 & $30 \mathrm{~s}$ \\
\hline Mechanical delay of SVR3 & $4 \mathrm{~s}$ \\
\hline Active power of DG & $1 \mathrm{MW}$ \\
\hline Reference voltage of DG $\left(\boldsymbol{V}_{\boldsymbol{r e f}}\right)$ & $7500 \mathrm{~V}$ \\
\hline Droop gain of DG $\left(\boldsymbol{K}_{\boldsymbol{q}(\boldsymbol{i})}\right.$ for $\left.\boldsymbol{i}=\{\mathbf{1}\}\right)$ & $0.5 \cdot 10^{-4} \mathrm{~V} / \mathrm{Var}$ \\
\hline
\end{tabular}

TABLE II

LOADS OF 8-BUS NETWORK

\begin{tabular}{|c|c|}
\hline Load & $Z_{L a} \rightarrow R=500 \Omega$ in parallel with $X=j 2000 \Omega$ \\
Bus 3 & $Z_{L b} \rightarrow R=500 \Omega$ in parallel with $X=j 2000 \Omega$ \\
& $Z_{L c} \rightarrow R=500 \Omega$ in parallel with $X=j 2000 \Omega$ \\
\hline Load & $Z_{L a} \rightarrow R=500 \Omega$ in parallel with $X=j 2000 \Omega$ \\
Bus 5 & $Z_{L b} \rightarrow R=500 \Omega$ in parallel with $X=j 2000 \Omega$ \\
& $Z_{L c} \rightarrow R=500 \Omega$ in parallel with $X=j 2000 \Omega$ \\
\hline Load & $Z_{L a} \rightarrow R=300 \Omega$ in parallel with $X=j 1200 \Omega$ \\
Bus 7 & $Z_{L b} \rightarrow R=300 \Omega$ in parallel with $X=j 1200 \Omega$ \\
& $Z_{L c} \rightarrow R=300 \Omega$ in parallel with $X=j 1200 \Omega$ \\
\hline Load & $Z_{L a} \rightarrow R=500 \Omega$ in parallel with $X=j 2000 \Omega$ \\
Bus 8 & $Z_{L b} \rightarrow R=500 \Omega$ in parallel with $X=j 2000 \Omega$ \\
& $Z_{L c} \rightarrow R=500 \Omega$ in parallel with $X=j 2000 \Omega$ \\
\hline
\end{tabular}

The tap positions and switching sequence for all SVRs of the 8-bus network obtained via the dynamic simulation of Simulink are presented in Fig. 2. It should be noted that since all the loads are balanced, the three phases of the SVRs undergo similar tap changes in this case. SVR 1 executes the first switching action after an intentional time delay $(10 \mathrm{sec})$, followed by subsequent switching actions with a mechanical time delay ( $2 \mathrm{sec}$ ), until the voltage lies within the bandwidth. Similarly, for SVR 2 and 3. Since SVR 1 has the lowest intentional and mechanical delay, it undergoes the highest number of tap changes. The final tap positions obtained via dynamic simulation for SVR 1, SVR 2 and SVR 3 is 11, 3, and 1 , respectively.

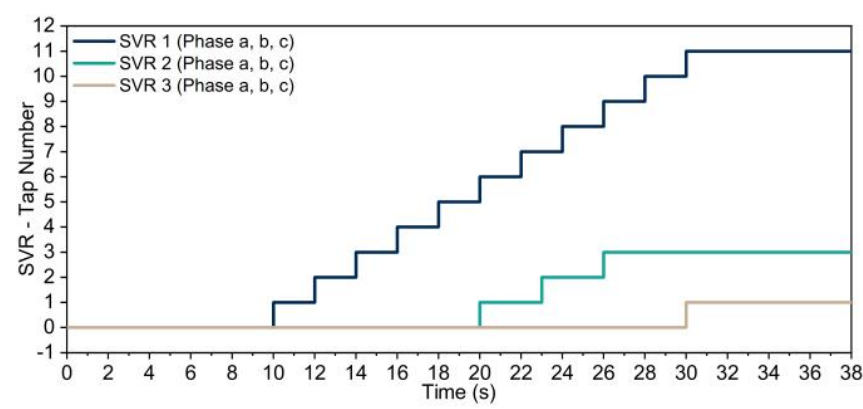

Fig. 2: Switching sequence of SVRs in the 8-Bus network versus time as calculated by MATLAB ${ }^{\circledR}$ Simulink.

In Figs. 3-6, the results of the SVR tap change versus iteration number are presented for the proposed algorithm as well as for the algorithms of [1] and [3]. Fig. 3 presents the tap change profile of all the SVRs versus the iteration number for the proposed algorithm without consideration of sensitivity parameters (refer Section IV of part I). In Fig. 4, the tap change profile versus iteration number is presented for the proposed algorithm with the consideration of the sensitivity parameters (refer Section VI of part I). The proposed algorithm, for both with and without sensitivity parameter, considers the actual reaction delays of LVCs. However, as observed in Fig. 4, the consideration of sensitivity parameters yields accelerated convergence. It is reminded that the proposed algorithm, both with and without sensitivity parameters, considers the same switching algorithm, thus, it presents identical results in both cases. Their main difference is that the usage of sensitivity parameters makes the execution of a complete power flow after each switching action unnecessary, reducing the total iteration number required for the final power flow solution. More details are provided in Part I.

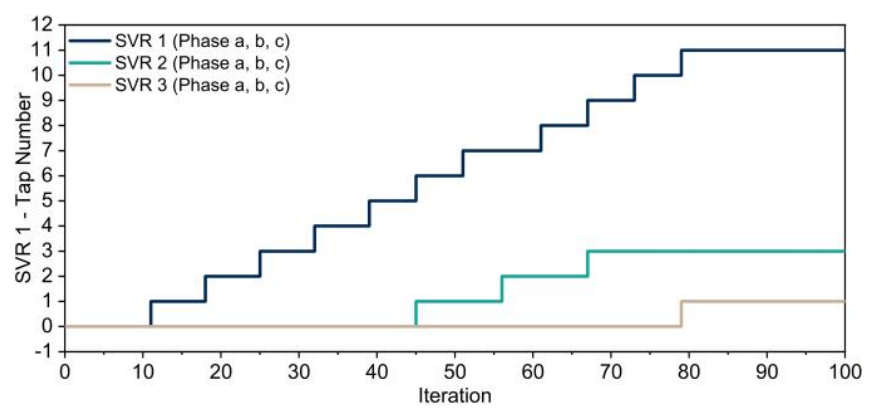

Fig. 3: Switching sequence of the three SVRs for the 8-Bus network versus iteration number, as calculated by the proposed method without sensitivity. 
"This work has been submitted to IEEE for possible publication. Copyright may be transferred without notice, after which this version may no longer be accessible."

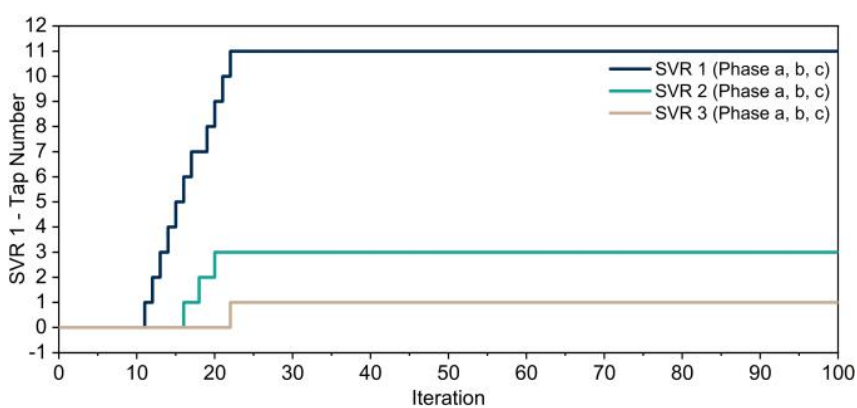

Fig. 4: Switching sequence of the three SVRs for the 8-Bus network versus iteration number, as calculated by the proposed method with sensitivity.

It is observed that the proposed power flow algorithm yields correct tap positions when compared to the dynamic simulation, both with and without sensitivities. This is due to the consideration of the actual switching sequence of LVCs, as presented in Part I Section IV. On the other hand, the LVC's states estimated by the power flow algorithms of [1] and [3] do not conform to the dynamic simulation, as shown in Figs. 5 and 6.

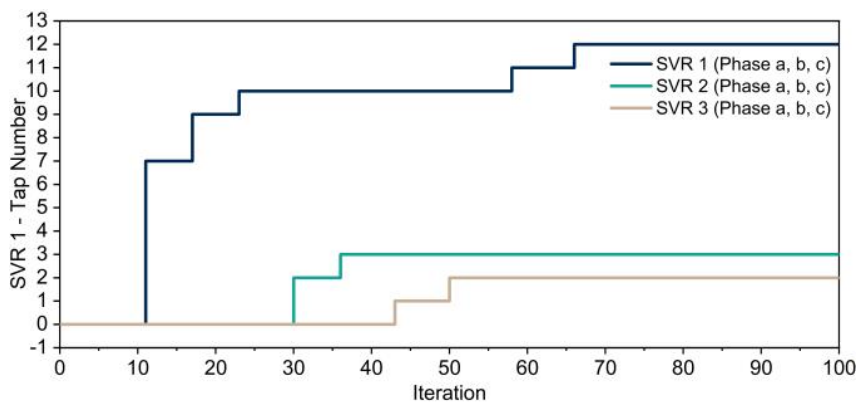

Fig. 5: Switching sequence of the three SVRs for the 8-Bus network versus iteration number, as calculated by the algorithm of reference [1].

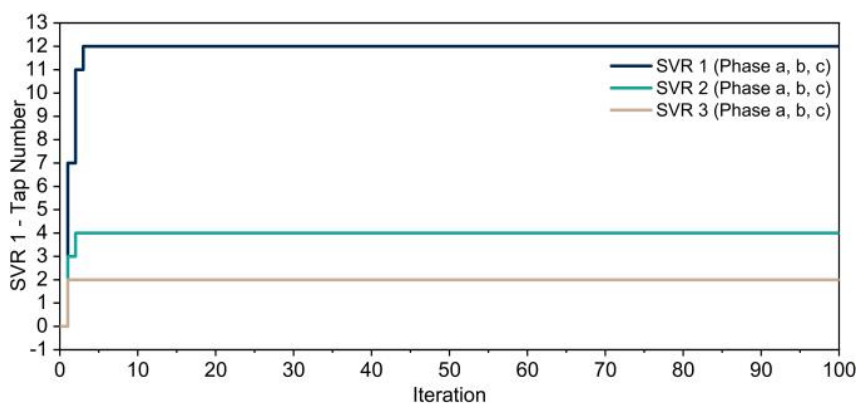

Fig. 6: Switching sequence of the three SVRs for the 8-Bus network versus iteration number, as calculated by the algorithm of reference [3].

Algorithm [1] is only able to correctly estimate the tap position of SVR 2 as shown in Fig 5. It is reminded that the authors in [1] divide the LVCs in delay groups based on their reaction delays. In this example, SVR 1, SVR 2 and SVR 3 belong to the first, second and third delay group, respectively. Initially, the SVR of the first delay group reacts, by varying its taps until its voltage lies inside the bandwidth. Subsequently, the SVR of the second delay group undertakes switching actions, and so on until all SVR voltages lie inside their bandwidths.

The algorithm of [3] reaches final tap position in only a few iterations (Fig. 6), because the algorithm completely neglects the LVC's reaction delays in its formulation, and thus, it updates all the LVC states simultaneously.
A detailed depiction of the convergence characteristic of the investigated algorithms is presented in Fig. 7. Figure 7 presents the maximum mismatch voltage error between two consecutive iterations in per-unit versus the iteration number. As observed, the proposed algorithm converges quickly to the correct solution when sensitivity parameters are considered. Although algorithm of [3] appears to have the fastest converge, the produced power flow results are not accurate compared to the dynamic simulation, as explained above. It is again highlighted here that although a difference in convergence of the proposed algorithm exists with and without the usage of the sensitivity parameters, the final results in both cases match those of the dynamic simulation.

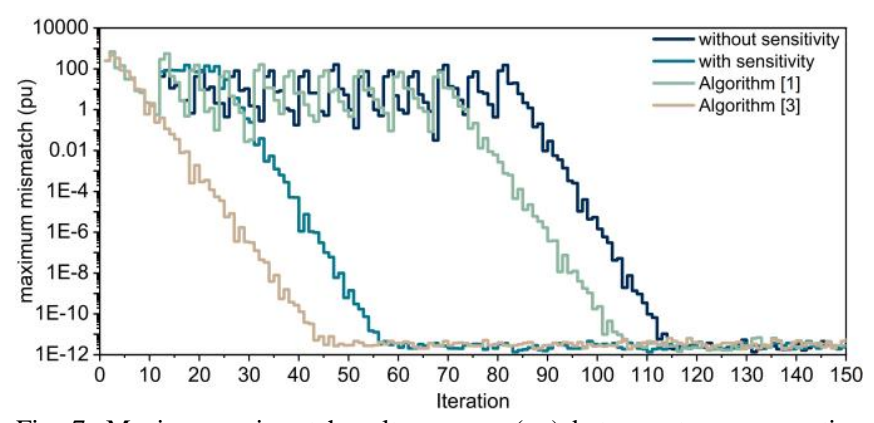

Fig. 7: Maximum mismatch voltage error (pu) between two consecutive iterations versus iteration number for all investigated algorithms.

In Fig. 8, the evolution of the voltage of bus 2 and 5 versus iteration is presented for the investigated methods. The proposed algorithm with and without the sensitivity yields the same final voltages but with different convergence speed as observed in Fig. 8. On the other hand, the algorithms of [1] and [3] yield different voltages, which are not in agreement with the proposed algorithm. The final voltages of the network for the investigated methods and Simulink are provided in Table III at the end of the paper. The proposed approach with and without sensitivities present almost identical results with those of Simulink, in contrast to the methods of [1] and [3] that show significant deviations.

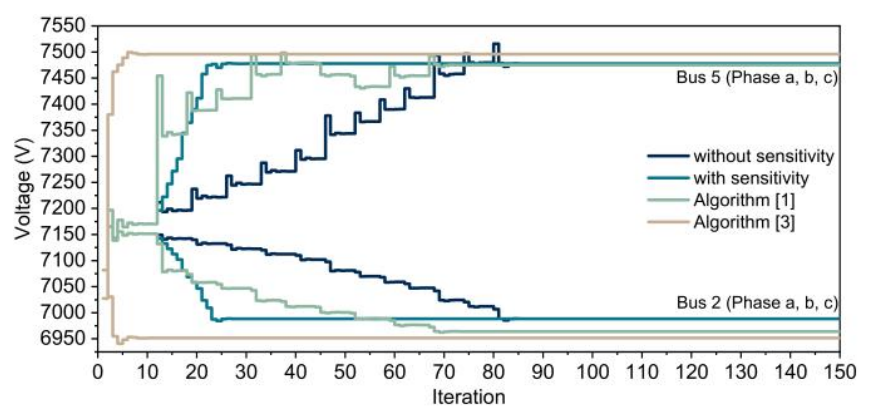

Fig. 8: Bus voltage evolution versus iteration for bus 2 and 5.

In Fig. 9, the total three-phase reactive power of the DG connected at bus 8 is presented. It operates in inductive mode according to droop equation (1), to mitigate the voltage rise caused by the high amount of generated active power [8]. It is observed that the proposed algorithm indicates a consumption of $542 \mathrm{kVar}$, which is exactly the same as in the dynamic simulation. Algorithms [1] and [3] yield different results. The deviation of the reactive power in algorithms [1] 
"This work has been submitted to IEEE for possible publication. Copyright may be transferred without notice, after which this version may no longer be accessible."

and [3] is caused due to the inaccurate state estimation of SVRs, which inevitably leads to an imprecise reactive power calculation.

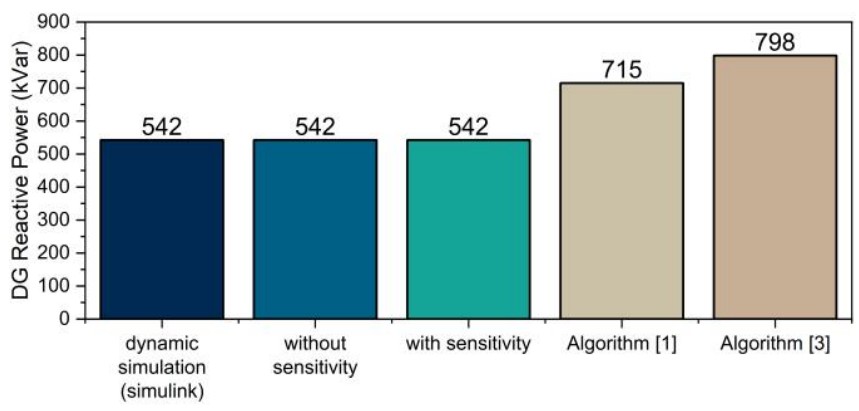

Fig. 9: Total reactive power consumption of the DG at bus 8 .

\section{B) 7-Bus Unbalanced Network}

An unbalanced 7-bus network consisting of an OLTC, a voltage-controlled capacitor, an SVR, and a DG is considered for further simulation and validation, as shown in Fig. 10. This network was selected for simulation since it includes all kind of LVCs (OLTC, SVR, Capacitor, DG), it is unbalanced and also its simple topology facilitates the comparison and interpretation of the simulation results. Moreover, its small size allows the execution of simulations in the time domain environment of MATLAB ${ }^{\circledR}$ Simulink.

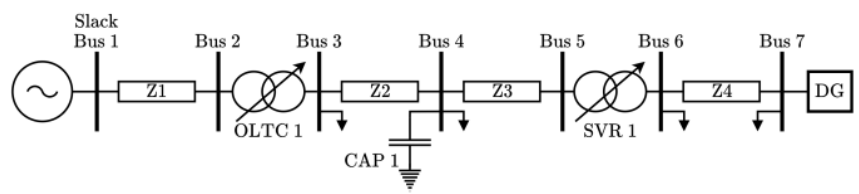

Fig. 10: 7-Bus unbalanced network consisting of one OLTC, one SVR, one three-phase capacitor and one DG.

The DG operates in $Q(V)$ droop control mode (see Eq. (1)). Data about the network, the controller of OLTC, SVR, capacitors and DG are presented in Table IV. The SVR is connected in wye configuration, while OLTC in $Y_{g}-Y_{g}$ connection [11, Section II.C]. Each phase of OLTC, SVR, and capacitor has its own local controller and is independently controlled. The DG generates balanced phaseto-neutral voltages [7]. The network supplies three balanced and one unbalanced three-phase constant impedance load, as shown in Table V.

Table VI summarizes the calculated LVC's states of each phase in the 7-Bus network using dynamic simulation, the proposed algorithm without and with sensitivity parameters, as well as the methods of [1] and [3]. It is observed that the proposed algorithm presents identical results with those of Simulink, confirming its accuracy. On the other hand, the calculated states of the approaches in [1], [3] deviate from those of Simulink. Moreover, Table VII at the end of the paper depicts indicatively the voltages of each phase for the last three buses of the network for the investigated approaches. The remaining buses are not depicted due to space limitation. As shown, the proposed method with and without sensitivity parameter yields near identical results with those of Simulink, while the other investigated power flow methods present significant deviations.

TABLE IV

PARAMETERS OF 7-BUS NETWORK

\begin{tabular}{|c|c|}
\hline Distance of the lines & $10 \mathrm{~km}$ \\
\hline Voltage of slack bus & $7200 \mathrm{~V}$ \\
\hline Frequency of the network & $50 \mathrm{~Hz}$ \\
\hline Resistance of lines & $0.4 \Omega / \mathrm{km}$ \\
\hline Self-reactance of the lines & $0.3 \Omega / \mathrm{km}$ \\
\hline Mutual-reactance of the lines & $0.1 \Omega / \mathrm{km}$ \\
\hline Reference voltage of SVR and OLTC & $7500 \mathrm{~V}$ \\
\hline Bandwidth of SVR and OLTC & $70 \mathrm{~V}$ \\
\hline Reference voltage of CAP & $7500 \mathrm{~V}$ \\
\hline Capacitance of each phase & $5 \cdot 10^{-6} \mathrm{~F}$ \\
\hline Bandwidth of Capacitors & $350 \mathrm{~V}$ \\
\hline Intentional delay of OLTC & $10 \mathrm{~s}$ \\
\hline Mechanical delay of OLTC & $2 \mathrm{~s}$ \\
\hline Intentional delay of CAP & $20 \mathrm{~s}$ \\
\hline Intentional delay of SVR & $30 \mathrm{~s}$ \\
\hline Mechanical delay of SVR & $4 \mathrm{~s}$ \\
\hline Active power of DG & $1 \mathrm{MW}$ \\
\hline Reference voltage of DG & $7500 \mathrm{~V}$ \\
\hline Droop gain of DG $(\boldsymbol{K}$ q $(\boldsymbol{i}) \boldsymbol{f o r} \boldsymbol{i}=\{\mathbf{1}\})$ & $0.5 \cdot 10^{-4} \mathrm{~V} / \mathrm{Var}$ \\
\hline
\end{tabular}

TABLE V

LOADS OF 7-BUS NETWORK

\begin{tabular}{|c|c|}
\hline Load & $Z_{L a} \rightarrow R=500 \Omega$ in parallel with $X=j 2000 \Omega$ \\
Bus 3 & $Z_{L b} \rightarrow R=500 \Omega$ in parallel with $X=j 2000 \Omega$ \\
& $Z_{L c} \rightarrow R=500 \Omega$ in parallel with $X=j 2000 \Omega$ \\
\hline Load & $Z_{L a} \rightarrow R=500 \Omega$ in parallel with $X=j 2000 \Omega$ \\
Bus 4 & $Z_{L b} \rightarrow R=250 \Omega$ in parallel with $X=j 1000 \Omega$ \\
& $Z_{L c} \rightarrow R=500 \Omega$ in parallel with $X=j 2000 \Omega$ \\
\hline Load & $Z_{L a} \rightarrow R=500 \Omega$ in parallel with $X=j 2000 \Omega$ \\
Bus 6 & $Z_{L b} \rightarrow R=500 \Omega$ in parallel with $X=j 2000 \Omega$ \\
& $Z_{L c} \rightarrow R=500 \Omega$ in parallel with $X=j 2000 \Omega$ \\
\hline Load & $Z_{L a} \rightarrow R=500 \Omega$ in parallel with $X=j 2000 \Omega$ \\
Bus 7 & $Z_{L b} \rightarrow R=500 \Omega$ in parallel with $X=j 2000 \Omega$ \\
& $Z_{L c} \rightarrow R=500 \Omega$ in parallel with $X=j 2000 \Omega$ \\
\hline
\end{tabular}

TABLE VI

LVC'S STATES CALCULATED BY THE INVESTIGATED APPROACHES FOR THE 7-BUS NETWORK

\begin{tabular}{|c|c|c|c|c|}
\cline { 2 - 5 } \multicolumn{1}{c|}{} & $\begin{array}{c}\text { OLTC 1 Taps } \\
(\mathbf{p h a s e} \mathbf{a}, \mathbf{b}, \mathbf{c})\end{array}$ & $\begin{array}{c}\text { CAP 1 } \\
\text { (phase a, b, c) }\end{array}$ & $\begin{array}{c}\text { SVR 1 Taps } \\
\text { (phase a, b, } \\
\mathbf{c})\end{array}$ & $\begin{array}{c}\text { DG Reactive } \\
\text { Power }\end{array}$ \\
\hline Simulink & $(10,12,10)$ & $(\mathrm{ON}, \mathrm{ON}, \mathrm{OFF})$ & $(2,4,3)$ & $\begin{array}{c}690 \mathrm{kVar} \\
\text { (inductive })\end{array}$ \\
\hline $\begin{array}{c}\text { without } \\
\text { sensitivity }\end{array}$ & $(10,12,10)$ & $(\mathrm{ON}, \mathrm{ON}, \mathrm{OFF})$ & $(2,4,3)$ & $\begin{array}{c}690 \mathrm{kVar} \\
\text { (inductive) }\end{array}$ \\
\hline $\begin{array}{c}\text { with } \\
\text { sensitivity }\end{array}$ & $(10,12,10)$ & $(\mathrm{ON}, \mathrm{ON}, \mathrm{OFF})$ & $(2,4,3)$ & $\begin{array}{c}690 \mathrm{kVar} \\
\text { (inductive) }\end{array}$ \\
\hline $\begin{array}{c}\text { Algorithm } \\
{[1]}\end{array}$ & $(11,10,10)$ & $(\mathrm{OFF}, \mathrm{ON}, \mathrm{OFF})$ & $(3,2,2)$ & $\begin{array}{c}666 \mathrm{kVar} \\
\text { (inductive) }\end{array}$ \\
\hline $\begin{array}{c}\text { Algorithm } \\
{[3]}\end{array}$ & $(11,11,10)$ & $(\mathrm{OFF}, \mathrm{ON}, \mathrm{OFF})$ & $(3,3,2)$ & $\begin{array}{c}625 \mathrm{kVar} \\
\text { (inductive })\end{array}$ \\
\hline
\end{tabular}

Finally, the number of iterations required by the algorithms to converge with an accuracy of $10^{-4} \mathrm{pu}$ are presented in Tables VIII and IX, for the 8-Bus and 7-Bus networks, respectively. As shown, the algorithm of [3] presents the fastest convergence since it calculates all LVC's state simultaneously ignoring their reaction delays. The proposed algorithm without the sensitivity parameters has the slowest convergence due to the successive power flow executions after each switching action. The proposed method with 
"This work has been submitted to IEEE for possible publication. Copyright may be transferred without notice, after which this version may no longer be accessible."

sensitivity parameters combines the high accuracy with the fast convergence, as no other power flow method so far.

TABLE VIII

REQUIRED ITERATIONS FOR THE INVESTIGATED APPROACHES IN THE 8-BUS NETWORK

\begin{tabular}{|c|c|c|c|c|}
\cline { 2 - 5 } \multicolumn{1}{c|}{} & $\begin{array}{c}\text { without } \\
\text { sensitivity }\end{array}$ & $\begin{array}{c}\text { with } \\
\text { sensitivity }\end{array}$ & $\begin{array}{c}\text { Algorithm } \\
{[1]}\end{array}$ & $\begin{array}{c}\text { Algorithm } \\
\text { [3] }\end{array}$ \\
\hline $\begin{array}{c}\text { Required } \\
\text { iterations }\end{array}$ & 74 & 27 & 61 & 9 \\
\hline
\end{tabular}

TABLE IX

REQUIRED ITERATIONS FOR THE INVESTIGATED APPROACHES IN THE 7-BUS NETWORK

\begin{tabular}{|c|c|c|c|c|}
\cline { 2 - 5 } \multicolumn{1}{c|}{} & $\begin{array}{c}\text { without } \\
\text { sensitivity }\end{array}$ & $\begin{array}{c}\text { with } \\
\text { sensitivity }\end{array}$ & $\begin{array}{c}\text { Algorithm } \\
\text { [1] }\end{array}$ & $\begin{array}{c}\text { Algorithm } \\
\text { [3] }\end{array}$ \\
\hline $\begin{array}{c}\text { Required } \\
\text { iterations }\end{array}$ & 96 & 29 & 76 & 8 \\
\hline
\end{tabular}

\section{INFLUENCE OF WEATHER ON THE LVC STATES AND POWER FLOW}

We conduct a case study on the large IEEE 8500-Node network to highlight the important impacts of weather on the LVC's state and power flow results, which cannot be investigated via conventional approaches. Moreover, the accuracy of the proposed sensitivity-based algorithm in a large-scale network is investigated.

\section{A) Network description}

Fig. 11 depicts the IEEE 8500-Node Network, which has been modified by including 4 DGs operating in several modes. The network originally includes 4 SVRs and 4 capacitors [9], as shown in Fig. 11.

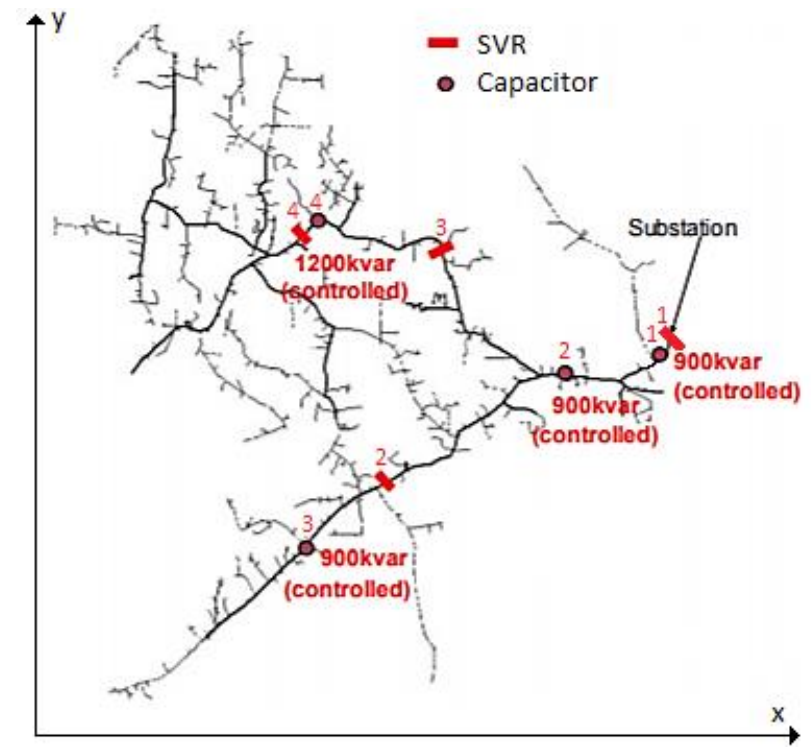

Fig. 11: IEEE 8500-Node network consisting of 4 SVRs and 4 three-phase capacitors [9].
To get a sense on the position of the buses in the network, the distance of each bus from the substation is depicted in Fig. 12 .

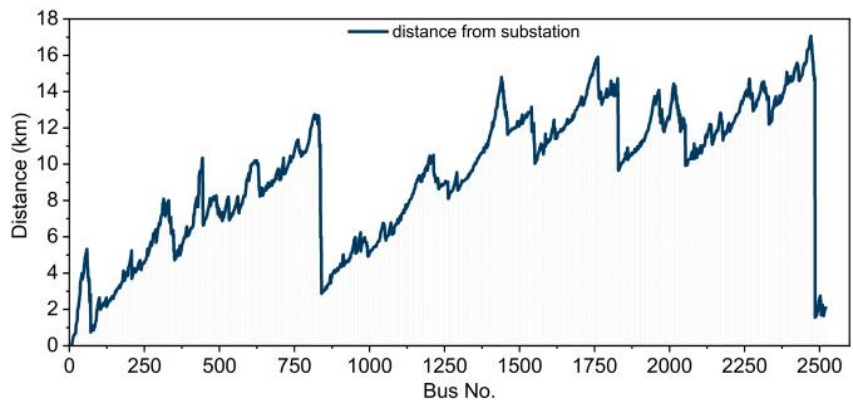

Fig. 12: Distance of the buses from the substation for the IEEE 8500-Node network.

The 4 additional DGs are connected to the buses 100, 350, 835,1600 . Data about the power profile and voltage/current profile of DGs are provided in Table X, while data about the reference voltages, the droop gains, the active powers, etc. are given in Table XI. For DG 1, which is a synchronous generator (SG), the negative- and zero-sequence impedance are $Z_{2}=Z_{0}=0.2+j 0.2$. DG 1 and DG 4 operate in constant voltage mode (treated as PV buses) and generate constant active power and positive-sequence voltage. DG 2 and DG 3 operate in droop control mode generating balanced currents (refer to Section III.D of Part I for more details about the operational modes of DGs).

Moreover, data of the LVCs are provided in Table XI. Each phase of the LVCs is independently controlled. The capacitors are voltage controlled and the SVRs are in wye configuration. The time delays of SVRs and capacitors are set based on their distance from the substation [6], while DGs react instantaneously. LVCs near to substation have faster reaction times as shown in Table XI. Each controller regulates the voltage of the connection point of LVC and no line drop compensator or any other remote voltage control are applied.

Finally, all the lines of the network were replaced with the Penguin ACSR [10]. It is a single-layer conductor with a cross-sectional area $125.1 \mathrm{~mm}^{2}$ composed of a 6 Aluminum and 1 steel wire. Penguin is the largest single-layer ACSR conductor and can successfully withstand the full load of the network in both investigated environmental conditions, without thermal violation. This modification was necessary in order to simulate the influence of weather and magnetic effects into the power flow results. With the original lines of IEEE 8500-node network, this would not be possible since all lines consist of constant impedances with unknown conductor specific details.

In Figs 13 and 14, the resistance and self-reactance of Penguin ACSR conductor are presented, respectively, as a function of conductor temperature and current. The methodology to calculate the resistance and self-reactance of Figs. 13 and 14 is presented in [7].

It is observed in Fig. 13 that the resistance of Penguin ACSR is strongly related to both current and conductor temperature (conductor temperature is a function of both current and weather conditions [7]). On the other hand, the self-reactance is more related to the current than the 
"This work has been submitted to IEEE for possible publication. Copyright may be transferred without notice, after which this version may no longer be accessible."

temperature. Nevertheless, the impact of weather is significant as demonstrated in the following subsection.

TABLE X

DG PARAMETERS FOR THE MODIFIED IEEE 8500-NODE NETWORK

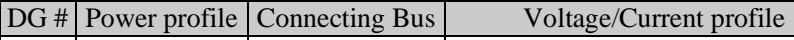

\begin{tabular}{|c|c|c|c|}
\hline 1 & Constant $\mathrm{PV}$ & 100 & SG (Unbalanced voltage and current) \\
\hline 2 & Droop $\mathrm{Q}(\mathrm{V})$ & 350 & Balanced Current \\
\hline 3 & Droop $\mathrm{Q}(\mathrm{V})$ & 835 & Balanced Current \\
\hline 4 & Constant $\mathrm{PV}$ & 1600 & Balanced Voltage \\
\hline
\end{tabular}

TABLE XI

PARAMETERS OF THE MODIFIED IEEE 8500-NODE NETWORK

\begin{tabular}{|c|c|}
\hline Length of the lines & Given in [13] \\
\hline Loads & Given in [13] \\
\hline Voltage of slack bus & $7200 \mathrm{~V}$ \\
\hline Frequency of the network & $50 \mathrm{~Hz}$ \\
\hline Line type and data & $\begin{array}{c}\text { All lines are Penguin ACSR [10]. } \\
\text { The mutual impedance between all } \\
\text { the lines was consider 0.1j Ohm/km. }\end{array}$ \\
\hline Reference voltage of SVRs & $7500 \mathrm{~V}$ \\
\hline Bandwidth of SVRs & $70 \mathrm{~V}$ \\
\hline Intentional delay of SVR 1 & $15 \mathrm{~s}$ \\
\hline Intentional delay of SVR 2 & $60 \mathrm{~s}$ \\
\hline Intentional delay of SVR 3 & $60 \mathrm{~s}$ \\
\hline Intentional delay of SVR 4 & $90 \mathrm{~s}$ \\
\hline Mechanical delay of SVRs & $2 \mathrm{~s}$ \\
\hline Intentional delay of CAP 1 & $30 \mathrm{~s}$ \\
\hline Intentional delay of CAP 2 & $45 \mathrm{~s}$ \\
\hline Intentional delay of CAP 3 & $75 \mathrm{~s}$ \\
\hline Intentional delay of CAP 4 & $75 \mathrm{~s}$ \\
\hline Reference voltage of CAPs & $7600 \mathrm{~V}$ \\
\hline Bandwidth of CAPs & $300 \mathrm{~V}$ \\
\hline Capacitance of each phase & $2 \cdot 10^{-5} \mathrm{~F}$ \\
\hline Active power of DGs & $2 \mathrm{MW}$ \\
\hline Reference voltage of DGs & $\left(\boldsymbol{V}_{\text {ref }}\right.$ ) \\
\hline Droop gain of DGs $\mathbf{2}$ \& 3 $\left(\boldsymbol{K}_{\boldsymbol{q}}\right)$ & $7500 \mathrm{~V}$ \\
\hline \multicolumn{2}{|c}{} \\
\hline
\end{tabular}

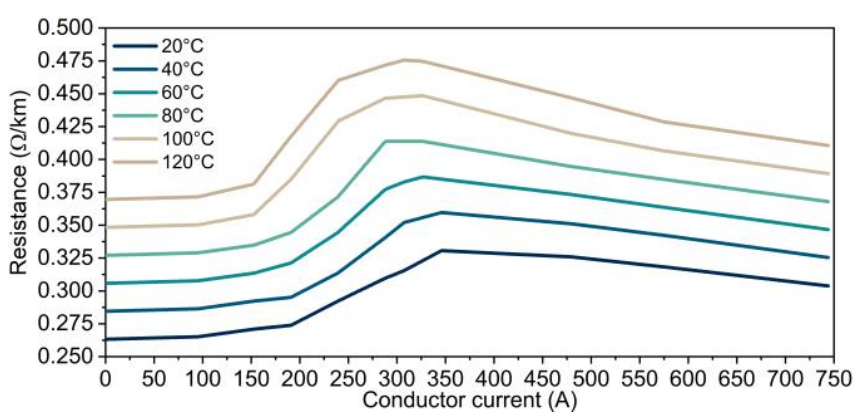

Fig. 13: Resistance of Penguin ACSR conductor, as a function of conductor temperature and current, considering weather and magnetic impacts.

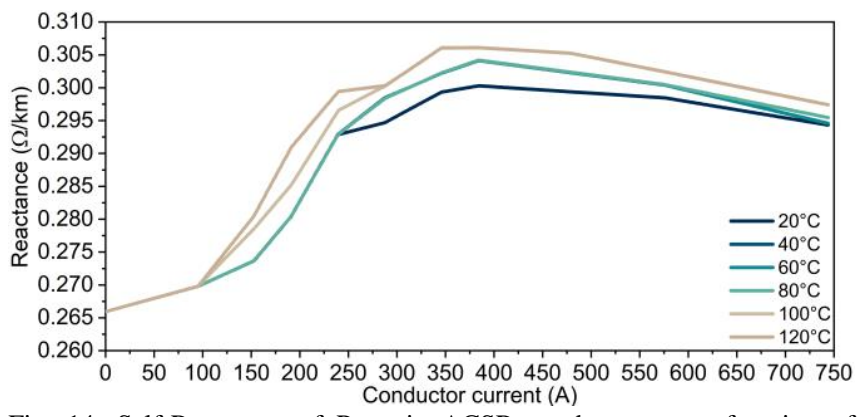

Fig. 14: Self-Reactance of Penguin ACSR conductor, as a function of conductor temperature and current, considering weather and magnetic impacts.

\section{B) Impacts of Weather on LVC's state estimation}

Simulation results and analysis are presented in this section. Simulations were undertaken in a generic $\mathrm{PC}$ with an Intel Core i7, 3.4GHz CPU and 16GB DDR3 RAM. As mentioned before, all investigated algorithms were coded in MATLAB $^{\circledR}$. Due to the large size of the network, modelling and dynamic simulations in Simulink are not possible here.

The case study involves simulation of two scenarios with two different weather conditions (summer and winter), as presented in Table XII. The summer condition corresponds to a typical summer day as measured at a weather station in Sindos (a suburb of Thessaloniki, Greece, with zero elevation) on 23/07/2018 at 14:00, while the second one corresponds to a typical winter night measured at the same station on 18/01/2018 at 21:00 [12].

TABLE XII

SUMMER AND WINTER WEATHER CONDITIONS

\begin{tabular}{|c|c|c|c|}
\cline { 2 - 4 } & $\begin{array}{c}\text { Ambient } \\
\text { temperature } \\
T_{a}\left({ }^{\circ} \mathrm{C}\right)\end{array}$ & $\begin{array}{c}\text { Solar irradiance } \\
q_{s}\left(\mathrm{~W} / \mathrm{m}^{2}\right)\end{array}$ & $\begin{array}{c}\text { Wind speed } \\
V_{w}(\mathrm{~km} / \mathrm{h})\end{array}$ \\
\hline Summer & 36.3 & 847 & 4.8 \\
\hline Winter & 7.3 & 0 & 14.5 \\
\hline
\end{tabular}

The directions of the lines were considered exactly as they are given in Fig. 11, while the wind direction was considered parallel to the $x$-axis of Fig. 11. The original network loads were considered for all buses [13]. In all approaches, tap position 2 was assumed as initial for all SVRs, while all the capacitors are initially considered OFF. As none of the algorithms other than the proposed are weather-dependent, the simulation results indicate that the proposed algorithm calculates accurate power flow results and LVC states considering the weather and magnetic effects, in contrast to the existing algorithms.

In Table XIII, the reactive power of the DGs in the IEEE 8500-node network is presented. As observed for the proposed algorithm, for the same loading condition, the summer and winter DG reactive powers are different due to the impact of weather and magnetic effects on the lines of the network. It should be noted that the proposed algorithm produces the same power flow results for the two cases, with and without sensitivity parameters. The difference observed between the two cases was in the convergence characteristics and the evolution of the solution states. On the other hand, Algorithms [1] and [3] do not consider any weather or magnetic effects and their results are not in agreement with the proposed algorithm. It is pointed out that when applying Algorithms [1] and [3], a constant impedance for all Penguin conductors of the network was considered equal to $\mathrm{Z}=0.4+0.3 \mathrm{j}(\mathrm{Ohm} / \mathrm{km})$. This value was calculated from Figs. 13 and 14 for a conductor current $\mathrm{I}=300 \mathrm{~A}$ and a temperature $\mathrm{T}=80^{\circ} \mathrm{C}$, which corresponds to the maximum conductor temperature [7].

The tap positions and switching sequence for SVR 3 in the modified IEEE 8500-node network are presented in Figs. 1517. Fig. 15 presents the tap positions and switching sequence for SVR 3 (all phases) obtained via the proposed algorithm, with and without the sensitivity parameters for the summer scenario. Fig. 16 presents the same for the winter scenario. In 
"This work has been submitted to IEEE for possible publication. Copyright may be transferred without notice, after which this version may no longer be accessible."

Fig. 17, the tap positions, and the switching sequence for SVR 3 (all phases) are presented for the Algorithms of [1] and [3].

As observed in Figs. 15 and 16, the final tap positions are in agreement for the proposed algorithm with and without the use of sensitivity parameters. This is because both cases use similar algorithms to estimate the exact switching sequence of SVRs and capacitors (see Section IV and VI of Part I). It is also observed that the use of sensitivity parameters yields a much faster solution. In summer, the final tap positions are $(7,6,5)$, while in winter they are $(6,5,4)$, for phase $a, b$, and $c$, respectively. This difference is caused due to the variation of line impedances as a result of the weather variation between summer and winter. This highlights the importance of considering the weather conditions into the power flow for more accurate analysis of power networks.

In Fig. 17, both algorithms [1] and [3] yield different results, which do not reflect the impact of weather and magnetic effects at all. As a result, the final tap positions obtained are not in agreement with the proposed algorithm. A summary of the final tap positions of the LVCs in the network is presented in Table XIV at the end of the paper.

The numbers of required iterations by the algorithms to converge with an accuracy of $10^{-4} \mathrm{pu}$ are presented in Table $\mathrm{XV}$ for the modified IEEE 8500-node network. Although algorithm [3] requires the lowest number of iterations, the final results are not accurate as they lack the consideration of the actual switching sequence of the LVCs. In addition, both algorithms [1] and [3] are not weather-dependent. As shown in Table XV, the proposed algorithm with the usage of sensitivity parameters appears to be the best power flow tool for networks with LCVs, since it combines high accuracy with fast convergence.

TABLE XIII

DG REACTIVE POWER FOR THE INVESTIGATED APPROACHES APPLIED IN

\begin{tabular}{|c|c|c|c|c|}
\cline { 2 - 5 } \multicolumn{1}{c|}{} & DG 1 & DG 2 & DG 3 & DG 4 \\
\hline $\begin{array}{c}\text { Proposed } \\
\text { Algorithm } \\
\text { (Summer) }\end{array}$ & $\begin{array}{c}3262 \mathrm{kVar} \\
\text { (capacitive) }\end{array}$ & $\begin{array}{c}570 \mathrm{kVar} \\
\text { (capacitive) }\end{array}$ & $\begin{array}{c}520 \mathrm{kVar} \\
\text { (inductive) }\end{array}$ & $\begin{array}{c}1780 \mathrm{kVar} \\
\text { (capacitive) }\end{array}$ \\
\hline $\begin{array}{c}\text { Proposed } \\
\text { Algorithm } \\
\text { (Winter) }\end{array}$ & $\begin{array}{c}2884 \mathrm{kVar} \\
\text { (capacitive) }\end{array}$ & $\begin{array}{c}499 \mathrm{kVar} \\
\text { (capacitive) }\end{array}$ & $\begin{array}{c}506 \mathrm{kVar} \\
\text { (inductive) }\end{array}$ & $\begin{array}{c}1542 \mathrm{kVar} \\
\text { (capacitive) }\end{array}$ \\
\hline $\begin{array}{c}\text { Algorithm } \\
\text { [1] }\end{array}$ & $\begin{array}{c}3728 \mathrm{kVar} \\
\text { (capacitive) }\end{array}$ & $\begin{array}{c}717 \mathrm{kVar} \\
\text { (capacitive) }\end{array}$ & $\begin{array}{c}572 \mathrm{kVar} \\
\text { (inductive) }\end{array}$ & $\begin{array}{c}1803 \mathrm{kVar} \\
\text { (capacitive) }\end{array}$ \\
\hline $\begin{array}{c}\text { Algorithm } \\
\text { [3] }\end{array}$ & $\begin{array}{c}3130 \mathrm{kVar} \\
\text { (capacitive) }\end{array}$ & $\begin{array}{c}660 \mathrm{kVar} \\
\text { (capacitive) }\end{array}$ & $\begin{array}{c}608 \mathrm{kVar} \\
\text { (inductive) }\end{array}$ & $\begin{array}{c}2518 \mathrm{kVar} \\
\text { (capacitive) }\end{array}$ \\
\hline
\end{tabular}

\section{C) Accuracy of the Sensitivity-Based Algorithm}

In this sub-section, we investigate how accurate the proposed sensitivity-based algorithm is, compared with the proposed algorithm without sensitivities, when applied in the large IEEE 8500-node network. Six scenarios were simulated for this purpose in the IEEE 8500-node network, explained below. In all scenarios, the winter condition was assumed for calculating the line impedances.

- Scenario 1: The loads of the network are reduced to $70 \%$ of their nominal value.

- Scenario 2: The loads of the network are increased to $120 \%$ of their nominal value.
- $\quad$ Scenario 3: The generated active power of each DG is increased to $3 \mathrm{MW}$.

- Scenario 4: The reference voltage of all SVRs, capacitors, and DGs is set to $7200 \mathrm{~V}$.

- Scenario 5: The initial tap position for all the SVRs is set to tap number 8 .

- Scenario 6: $K_{q(i)}$ for $i=\{1,2,3,4\}$ is set to 1 . It practically means that the DGs generate almost zero reactive power.

The results of LVC's state are presented in Table XVI for the proposed method without and with the use of sensitivity. As shown, the algorithms produce identical results in the first four and $6^{\text {th }}$ scenario. In most of the simulations presented in this manuscript, there has been full agreement between the proposed algorithm without and with sensitivities. However, a slight difference is observed in the state estimation of SVR 4 of scenario 5 (highlighted in bold in Table XVI), where the two algorithms output $(3,2,0)$ and $(3,1,1)$, respectively. This difference is attributed to the fact that the usage of sensitivities involves predictor and corrector steps, which are approximations. Nevertheless, this difference is negligible in comparison to the major reduction in the number of iterations required to obtain the power flow solution when sensitivities are adopted, as observed in the last column of Table XVI.

\section{CONCLUSION}

This part II presents simulation results of the proposed sensitivity-based three-phase weather-dependent power flow method presented in Part I. The proposed algorithm accurately calculates the power flow and estimates the LVC states in distribution networks, by considering the actual switching sequence of LVCs, based on their intentional and mechanical time delays. The proposed algorithm is validated through dynamic simulation in MATLAB ${ }^{\circledR}$ Simulink. Based on the simulation results, the following three general conclusions are derived: a) the proposed method presents more precise power flow results and LVC's state estimation than the existing methods, b) the weather variations affect the LVC's state estimation in distribution networks, thus they should be considered for a more precise analysis, c) the usage of sensitivity parameters significantly accelerates the convergence of the algorithm, and therefore, the computation time. The last property is very important in real-time distribution management system applications, where the power flow is required to be executed many times in a short time period.

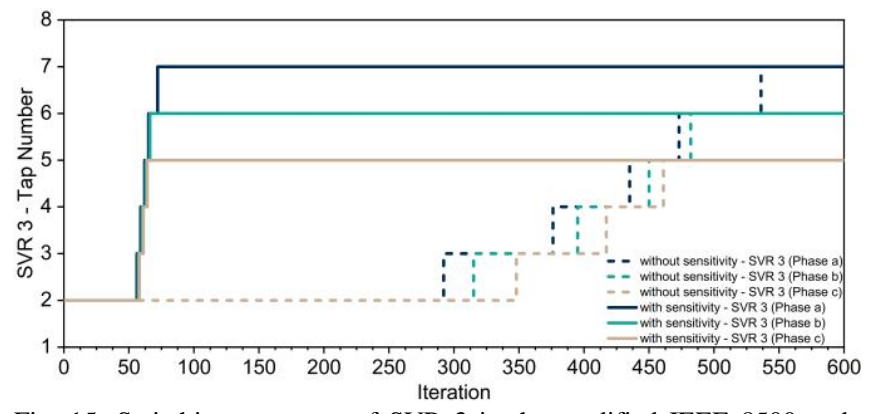

Fig. 15: Switching sequence of SVR 3 in the modified IEEE 8500-node network for summer obtained via the proposed algorithm. 
"This work has been submitted to IEEE for possible publication. Copyright may be transferred without notice, after which this version may no longer be accessible."

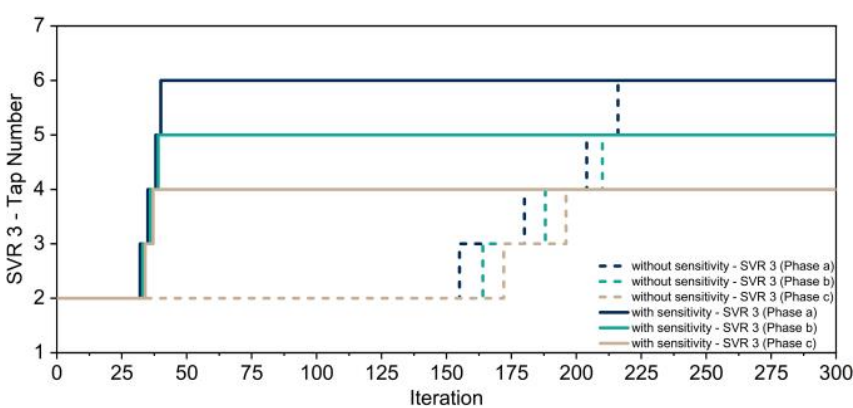

Fig. 16: Switching sequence of SVR 3 in the modified IEEE 8500-node network for winter obtained via the proposed algorithm.

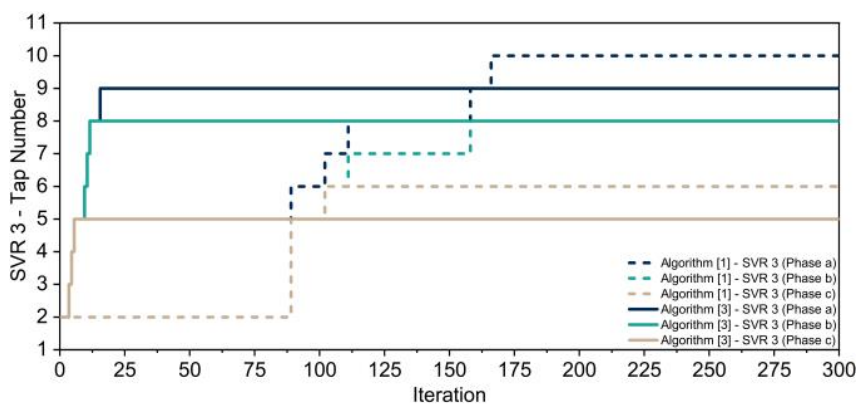

Fig. 17: Switching sequence of SVR 3 in the modified IEEE 8500-node network obtained via algorithm of [1] and [3].

TABLE XV

REQUIRED ITERATIONS FOR THE INVESTIGATED APPROACHES APPLIED IN THE MODIFIED IEEE 8500-NODE

\begin{tabular}{|c|c|}
\cline { 2 - 2 } \multicolumn{1}{c|}{} & Required iterations \\
\hline Proposed-without sensitivity (summer) & 553 \\
\hline Proposed-with sensitivity (summer) & 79 \\
\hline Proposed-without sensitivity (winter) & 261 \\
\hline Proposed-with sensitivity (winter) & 49 \\
\hline Algorithm [1] & 182 \\
\hline Algorithm [3] & 21 \\
\hline
\end{tabular}

\section{REFERENCES}

[1] I. Roytelman and V. Ganesan, "Modeling of local controllers in distribution network applications," IEEE Trans. Power Del., vol. 15, no. 4, pp. 1232-1237, Oct. 2000.

[2] R. A. Jabr, I. Džafic, S. Karaki, "Tracking Transformer Tap Position in Real-Time Distribution Network Power Flow Applications", IEEE Trans. Smart grid, Vol. 9, No. 4, 2018

[3] I. Džafic, R. A. Jabr, E. Halilovic, and B. C. Pal, "A sensitivity approach to model local voltage controllers in distribution networks," IEEE Trans. Power Syst., vol. 29, no. 3, pp. 1419-1428, May 2014.

[4] IEEE Standard for Interconnection and Interoperability of Distributed Energy Resources with Associated Electric Power Systems Interfaces, IEEE Std 1547-2018, Feb. 2018.

[5] M. Yorino et al., "An optimal autonomous decentralized control method for voltage control devices by using a multi-agent system", IEEE Trans. Power Syst., Vol. 30, No. 5, pp. 2225-2233, 2015.

[6] Don Wareham, "Step Voltage Regulators", Cooper Power Systems by Eaton, 2013, Accessed on: November 1, 2020. [Online].Available: http://www.cscos.com/wp-content/uploads/NY1839-EatonRegulators-D.Wareham.pdf

[7] E. Pompodakis, A. Ahmed, M. Alexiadis, "A Three-Phase WeatherDependent Power Flow Approach for 4-Wire Multi-Grounded Unbalanced Microgrids with Bare Overhead Conductors", IEEE Transactions on Power Systems (Early Access), DOI:10.1109/TPWRS.2020.3029053, October 2020

[8] Kraiczy et. al., "Parallel Operation of Transformers With on Load Tap Changer and Photovoltaic Systems With Reactive Power Control", IEEE Trans. Smart Grid, Vol. 9, No. 6, pp. 6419-6428, 2018.

[9] R. F. Arritt, R. C. Dugan, "The IEEE 8500-node test feeder", Proc. IEEE Transmiss. Distrib. Conf. Expo., pp. 1-6, Apr. 2010.IEEE PES (2020, March.) Distribution test feeders, "The IEEE European Low Voltage Test Feeder". [Online]. Available: http://www.ewh.ieee.org/soc/pes/dsacom/testfeeders/index.html

[10] DICABS Conductors Technical Catalogue, "Online Catalog", Accessed on: February 25, 2020. [Online].Available: https://www.academia.edu/34442747/Conductor_Technical_Catalogu re

[11] I. Huseinagić, I. Džafić, R. A. Jabr, “A compensation technique for unsymmetrical three-phase power flow", Int. Symposium. Ind Electron. (INDEL), Bosnia, 3-5 November, 2016.

[12] K. Lagouvardos et. al, "The automatic weather stations NOANN network of the National Observatory of Athens: operation and database", Geoscience Data Journal, Vol. 4, No. 1, pp. 4-16, 2017.

[13] IEEE PES. (2020, December.) Distribution test feeders, "8500-Node Test Feeder". [Online]. Available:

http://www.ewh.ieee.org/soc/pes/dsacom/testfeeders/index.html

TABLE III

Voltage PROFILE OF THE INVESTIGATEd METHOdS FOR THE 8-Bus BALANCED NETWORK Node 2a (V) Node 3a (V) Node 4a (V) Node 5a (V) Node 6a (V) Node 7a (V) Node 8a (V)

\begin{tabular}{|c|c|c|c|c|c|c|c|}
\hline Simulink & 6988.3309 & 7468.7787 & 7340.1399 & 7477.7693 & 7421.3890 & 7467.8162 & 7527.2533 \\
\hline without sensitivity & 6988.3417 & 7468.7902 & 7340.1411 & 7477.7687 & 7421.3933 & 7467.7770 & 7527.1493 \\
\hline with sensitivity & 6988.3417 & 7468.7902 & 7340.1411 & 7477.7687 & 7421.3933 & 7467.7770 & 7527.1493 \\
\hline Algorithm [1] & 6963.9601 & 7486.2571 & 7337.4348 & 7475.0117 & 7400.0861 & 7492.5872 & 7535.7599 \\
\hline Algorithm [3] & 6951.5222 & 7472.8864 & 7313.0733 & 7495.9002 & 7412.0259 & 7504.6762 & 7539.9454 \\
\hline
\end{tabular}

TABLE VII

VOLTAGE PROFILE (OF THE LAST THREE BUSES) OF THE INVESTIGATED METHODS FOR THE 7-BUS UNBALANCED NETWORK Node 5a (V) Node 5b (V) Node 5c (V) Node 6a (V) Node 6b (V) Node 6c (V) Node 7a (V) Node 7b (V) Node 7c (V)

\begin{tabular}{|c|c|c|c|c|c|c|c|c|c|}
\hline Simulink & 7391.8685 & 7311.9286 & 7350.1776 & 7484.2669 & 7494.7264 & 7488.0011 & 7534.5769 & 7534.5769 & 7534.5769 \\
\hline with $\mathrm{s}$ & 7391 & 731 & 735 & 748 & 749 & 74 & 995 & 095 & 095 \\
\hline Algorithm [1] & 7340.9089 & 7412.7342 & 7381.0320 & 7478.5510 & 7505.3934 & 7473.2949 & 7533.3146 & 7533.3146 & 7533.3146 \\
\hline Algorithm [3] & 7348.0466 & 7337.2315 & 7385.8412 & 7485.8225 & 7474.8046 & 7478.1643 & 7531.2463 & 7531.2463 & 7531.2463 \\
\hline
\end{tabular}


"This work has been submitted to IEEE for possible publication. Copyright may be transferred without notice, after which this version may no longer be accessible."

TABLE XIV

LVC'S STATES CALCULATED BY THE INVESTIGATED APPROACHES FOR THE MODIFIED IEEE 8500-NODE

\begin{tabular}{|c|c|c|c|c|c|c|c|c|}
\hline & \begin{tabular}{|c|} 
SVR 1 \\
(phase a, b, c)
\end{tabular} & $\begin{array}{c}\text { SVR 2 } \\
\text { hase a, b, c }\end{array}$ & $\begin{array}{c}\text { SVR 3 } \\
\text { ohase a, b, }\end{array}$ & $\begin{array}{c}\begin{array}{c}\text { SVR 4 } \\
\text { phase a, b, c) }\end{array} \\
\end{array}$ & $\begin{array}{c}\text { CAP 1 } \\
(\text { phase a, b, c) }\end{array}$ & $\begin{array}{c}\text { CAP 2 } \\
(\text { phase a, b, c) }\end{array}$ & $\begin{array}{c}\text { CAP 3 } \\
\text { (phase a, b, c) }\end{array}$ & $\begin{array}{c}\text { CAP 4 } \\
(\text { phase a, b, c) } \\
\end{array}$ \\
\hline ner & $(6,6,6)$ & $(1,2,2)$ & $(7,6,5)$ & $(3,2,2)$ & (OFF, OFF, OFF) & $(\mathrm{ON}, \mathrm{ON}, \mathrm{ON})$ & $\mathrm{DFF}, \mathrm{OFF}, \mathrm{OF}$ & 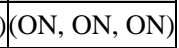 \\
\hline Prop & & & & & F) & & & \\
\hline & $(6,6$ & & $(10$ & & (OFF, & N, & UTF & d(UN \\
\hline [3] & $(6,6,6)$ & $(1,2,2)$ & $(9,8,5)$ & $(4,3,2)$ & $(\mathrm{ON}, \mathrm{ON}, \mathrm{ON})$ & $\mathrm{ON}, \mathrm{ON}, \mathrm{C}$ & (OFF, O & $(\mathrm{ON}$ \\
\hline
\end{tabular}

TABLE XVI

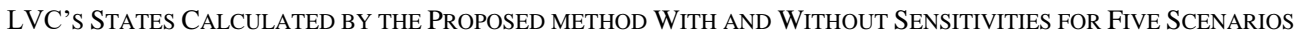

\begin{tabular}{|c|c|c|c|c|c|c|c|c|c|c|}
\hline & & \begin{tabular}{|c|} 
SVR 1 \\
$($ phase a, b, c)
\end{tabular} & \begin{tabular}{|c|} 
SVR 2 \\
$($ phase a, b, c c)
\end{tabular} & $\begin{array}{c}\text { SVR 3 } \\
(\text { phase a, b, c) }\end{array}$ & $\begin{array}{c}\text { SVR 4 } \\
(\text { phase a, b, c) }\end{array}$ & $\begin{array}{c}\text { CAP 1 } \\
(\text { phase a, b, c) }\end{array}$ & $\begin{array}{c}\text { CAP 2 } \\
(\text { phase a, b, c) }\end{array}$ & $\begin{array}{c}\text { CAP 3 } \\
(\text { phase a, b, c) }\end{array}$ & $\begin{array}{c}\text { CAP 4 } \\
(\text { phase a, b, c) }\end{array}$ & atic \\
\hline \multirow{2}{*}{$\begin{array}{c}\text { Scenario } \\
1\end{array}$} & without sensitivity & $(6,6,6)$ & $(0,1,1)$ & $(3,3,3)$ & $(2,2,2)$ & (OFF, OFF, OFF) & (OFF, OFF, OFF) & (OFF, OFF, OFF) & $(\mathrm{ON}, \mathrm{ON}, \mathrm{ON})$ & 143 \\
\hline & with sensitivity & $(6,6,6)$ & $(0,1,1)$ & 3) & 2) & (OFF, OFF, OFF) & (OFF, OFF, OFF) & (OFF, OFF, OFF) & $(\mathrm{ON}, \mathrm{ON}, \mathrm{ON})$ & 34 \\
\hline \multirow{2}{*}{$\begin{array}{c}\text { Scenario } \\
2\end{array}$} & without sensitivity & 7) & 21 & $(14$, & 2) & (OFF, & $(\mathrm{ON}$ & $(\mathrm{OFF}$, & $(\mathrm{ON}$, & 773 \\
\hline & with sensitivity & 7) & $(3$, & $(14$, & 2) & (OFF, OFF, OFF) & $(\mathrm{ON}, \mathrm{ON}, \mathrm{ON})$ & (OFF, OFF, OFF) & $(\mathrm{ON}, \mathrm{O}$ & 109 \\
\hline \multirow{2}{*}{$\begin{array}{c}\text { Scenario } \\
3\end{array}$} & without sensitivity & 6) & -7 & , (3) & 2) & F) & F) & (OFH & $(\mathrm{ON}$ & 17 \\
\hline & माiा & $(6$ & (1) & (2) & 2) & $\mathrm{OFF})$ & (OFF & (OFF, OFF, OFF) & $(\mathrm{ON}, \mathrm{C}$ & 19 \\
\hline \multirow{2}{*}{$\begin{array}{c}\text { Scenario } \\
4\end{array}$} & without sensitivity & $(0,0,0)$ & $(1,2,2)$ & $(8,5,4)$ & $(4,2,2)$ & (OFF, OFF, OFF) & (OFF, OFF, OFF) & (OFF, OFF, OFF) & (ON, OFF, OFF) & 169 \\
\hline & with sensitivity & $(0,0,0)$ & $(1,2,2)$ & $(8,5,4)$ & $(4,2,2)$ & (OFF, OFF, OFF) & (OFF, OFF, OFF) & (OFF, OFF, OFF) & $(\mathrm{ON}, \mathrm{OFF}, \mathrm{OFF})$ & 38 \\
\hline \multirow{2}{*}{$\begin{array}{c}\text { Scenario } \\
5\end{array}$} & without sensitivity & $(7,7,7)$ & $(1,1,1)$ & $(8,4,2)$ & $(3,2,0)$ & (OFF, OFF, OFF) & $(\mathrm{ON}, \mathrm{ON}, \mathrm{ON})$ & (OFF, OFF, OFF) & $(\mathrm{ON}, \mathrm{ON}, \mathrm{ON})$ & 871 \\
\hline & with sensitivity & $(7,7,7)$ & $(1,1,1)$ & $(8,4,2)$ & $(3,1,1)$ & (OFF, OFF, OFF) & $(\mathrm{ON}, \mathrm{ON}, \mathrm{ON})$ & (OFF, OFF, OFF) & $(\mathrm{ON}, \mathrm{ON}, \mathrm{ON})$ & 116 \\
\hline \multirow{2}{*}{$\begin{array}{c}\text { Scenario } \\
6\end{array}$} & without sensitivity & $(6,6,6)$ & 3) & $(9$, & $(3,2,2)$ & $(\mathrm{ON}, \mathrm{ON}, \mathrm{ON})$ & $(\mathrm{ON}, \mathrm{ON}, \mathrm{ON})$ & (OFF, OFF, OFF) & $(\mathrm{ON}, \mathrm{ON}, \mathrm{ON})$ & 379 \\
\hline & with sensitivity & $(6,6,6)$ & $(2,3,3)$ & $(9,8,7)$ & $(3,2,2)$ & $(\mathrm{ON}, \mathrm{ON}, \mathrm{ON})$ & $(\mathrm{ON}, \mathrm{ON}, \mathrm{ON})$ & (OFF, OFF, OFF) & $(\mathrm{ON}, \mathrm{ON}, \mathrm{ON})$ & 70 \\
\hline
\end{tabular}

